\title{
Le retard mental de la trisomie 21 serait dû à la présence d'un seul gène en triple dose
}

Dans le chromosome 21, la région critique responsable de la débilité mentale chez le sujet trisomique est limitée à $2 \mathrm{lq} 22.2$, et ne s'étend que sur 2 mégabases, ce qui est peut-être beaucoup, mais peu comparativement à la totalité du chromosome. Cette région a donc fait l'objet d'une recherche intense. En effet, si le retard mental de la trisomie 21 n'était dû qu'à un seul gène, les espoirs de thérapie génique pour cette maladie deviendraient les mêmes que pour les maladies mendéliennes.

Or, il existe, dans cette région 2 lq22.2, un gène codant pour une protéine kinase [1] qui a rapidement suscité un grand intérêt en raison de son analogie avec le gène minibrain (ou $m n b$ ) de la drosophile. Comme son nom le laisse deviner, les mutations avec perte de fonction de $m n b$ entraînent une réduction considérable du cerveau des souris [2]. Le gène humain $(M N B)$ est exprimé dans le cerveau adulte, ainsi que dans de nombreux tissus [3] ; il semble bien conservé au cours de l'évolution puisqu'on trouve, chez la souris et le rat, des gènes homologues presque identiques au gène humain (99\% d'identité pour la protéine présomptive) [4]. Le gène de la souris est localisé sur le chromosome 16 , homologue partiel du chromosome 21 humain. En outre, l'analyse de l'expression temporo-spatiale chez l'embryon de souris (avec signaux abondants dans le cerveau, la moelle épinière et la rétine) est compatible avec les manifestations phénotypiques du "mongolisme». Ce faisceau d'éléments encourageait à penser que $M N B$ jouait un rôle essentiel dans la pathogénie de la trisomie
21. Il fallait donc aller plus loin et reproduire le modèle chez l'animal afin de voir s'il entraînait, en triple dose, des troubles de l'apprentissage. Pour y parvenir, un groupe américain a eu recours à une technique originale en créant une "bibliothèque vivante" de souris transgéniques [5]. Quatre YAC (yeast artificial chromosome), en bibliothèque contiguë, contenaient la totalité des deux mégabases de la région critique. Des souris transgéniques furent créées avec chacun d'eux puis soumises à une série de tests conçus pour mesurer leurs performances d'apprentissage. L'un d'eux, le labyrinthe aquatique de Morris, consiste à habituer les souris à nager dans une piscine où se trouve une plateforme immergée qui leur permet de se reposer. Puis, la plate-forme étant retirée, un classement peut être effectué entre les souris explorant avec insistance le lieu où se trouvait précédemment la plate-forme et celles qui nagent n'importe où [6]. Comparativement aux souris témoins ou ayant reçu les autres YAC, les souris ayant reçu le YAC contenant $M N B$ ont à ces tests des performances nettement plus mauvaises. Pour affiner encore la recherche, on réalisa une fragmentation de ce YAC de $570 \mathrm{~kb}$ et les souris transgéniques contenant le fragment (de $180 \mathrm{~kb}$ ) dans lequel $M N B$ était encore présent, eurent des résultats tout aussi médiocres. L'étude histopathologique révéla aussi, chez les souris possédant le YAC de $570 \mathrm{~kb}$ - et malgré leur déficience mentale - une nette augmentation de la densité des neurones corticaux. Cette étude, réalisée avec le plus grand soin, avec vérification de l'intégrité et du nombre de copies des transgènes, et tenant compte du sexe des souris hybrides Fl pour pallier des effets épigénétiques comme l'empreinte parentale, est fort intéressante à plus d'un titre. La stratégie de "bibliothèque in vivo" adoptée ici permet d'identifier rapidement, dans une grande région à partir d'un contig de YAC, un gène et sa fonction et peut avoir de nombreuses autres applications en pathologie humaine. Pour ne citer qu'un exemple, la recherche d'oncogènes dans des régions fréquemment amplifiées dans les cellules tumorales - comme la région 1q32.4 dans le cancer du sein - pourrait ainsi être grandement facilitée [7]. Mais surtout, comme le fragment de $180 \mathrm{~kb}$ ne doit guère contenir que le gène $M N B$, celui-ci a de fortes chances d'être le gène responsable de la débilité mentale dans la trisomie 21. Cette découverte, si elle est confirmée, sera d'une portée considérable puisque la trisomie 21 , cause majeure des débilités mentales chez l'homme, et jusqu'alors prototype des maladies par aberration chromosmique, en serait presque réduite à une maladie moléculaire: son phénotype serait dû à une triple dose de quelques gènes contigus de la région 21 q22. En outre, si le gène $M N B$ a des conséquences sur l'apprentissage et la mémoire quand il est en triple dose, il pourrait bien, en simple dose, être aussi responsable des certains retards mentaux (peut-être avec microcéphalie ?). D'où l'intérêt de rechercher délétions et mutations suivies de perte de fonction de $M N B$ chez des sujets dont le retard mental est resté jusqu'alors inexpliqué.

S.G. 
1. Patil N, Cox DR, Bhat D, Faham M, Myers RM Peterson AS. A potassium channel mutation in weaver mice implicates membrane excitability in granule cell differentiation. Nature Genet 1995 11: 126-9

2. Tejedor F, Zhu XR, Kaltenbach E, Ackermann A, Baumann A, Canal I, Heisenberg M, Fischbach KF, Pongs O. Minibrain: a new protein kinase family involved in postembryonic neurogenesis in Drosophila. Neuron 1995; 14 : 287-301.

3. Shindoh N, Kudoh J, Maeda H, Yamaki A,
Minoshima S, Shimizu Y, Shimizu N. Cloning of a human homolog of the Drosophila minibrain/rat Dyrk gene from the Down syndrome critical region of chromosome 21. Biochem Biophys Res Commun 1996; 225 : 92-9.

4. Song WJ, Sternberg LR, Kasten-Sportès C, van Keuren ML, Chung SL, et al. Isolation of human and murine homologs of the Drosophila minibrain gene; human homolog maps to chromosome $2 \operatorname{lq} 22.2$ in the Down syndrome critical region. Genomics 1996; 38: 331-9.

5. Smith DJ, Stevens ME, Sudanagunta SP, Bron- son RT, Makhinson M, Watabe AM, O'Dell T], Fung J, Weier HUG, Cheng JF, Rubin EM. Func tional screening of $2 \mathrm{Mb}$ of human chromosome 21 q.22.2 in transgenic mice implicates minibrain in learning defects associated with Down syndrome. Nature Genet 1997; $16: 28-35$

6. Silva AJ, Paylor R, Wehner JM, Tonegawa S Impaired spatial learning in alpha-calcium-calmodulin kinase II mutant mice. Science 1992; 257 206-11.

7. Kola I. Simple minded mice from in vivo librairies. Nature Genet 1997; $16: 8-9$

\section{BRÈVES}

Des souris transgéniques possédant des fragments de chromosomes humains fonctionnels. Les techniques de transgenèse chez la souris butent souvent sur la taille des fragments génétiques qu'il faut transférer pour obtenir une expression qualitativement et quantitativement satisfaisante du transgène. Cependant, des progrès ont été réalisés récemment grâce au transfert de très grands fragments sous la forme de chromosomes artificiels de levure, isolés ou combinés $\left(\mathrm{m} / \mathrm{s} n^{\circ} 4\right.$, vol.9, p. 477). C'est ainsi qu'ont pu être obtenues des souris possédant les locus de la $\beta$-globine et des immunoglobulines humaines $(\mathrm{m} / \mathrm{s}$ $n^{\circ} 1$, vol. 12 , p. 103). Avec ces méthodes, cependant, la taille maximale de l'ADN transféré reste limitée à un maximum de l'ordre de la mégabase. C'est la raison pour laquelle une équipe japonaise a testé une méthode permettant de transférer des chromosomes entiers. La base de cette technique est le transfert dans des cellules souches embryonnaires (cellules ES) de chromosomes par l'intermédiaire de microcellules (MMCT, microcellmediated chromosome transfer). Cette approche consiste à fusionner des fibroblastes humains avec des cellules d'une lignée murine afin d'obtenir des hybrides somatiques contenant chacun au moins un gène marqueur de résistance à l'antibiotique G418 intégré dans un chromosome humain. Ensuite, un choc hypotonique permet de faire éclater les hybrides en "microcellules" contenant chacune, en moyenne, un chromosome humain. Ces micro-cellules sont alors fusionnées à nouveau avec des cellules murines qui sont ensuite sélectionnées par le G418 et la nature des chromosomes contenus par ces hybrides de deuxième génération est analysée par PCR et par hybridation in situ. La dernière étape consiste à obtenir à nouveau, à partir des clones d'hybrides somatiques de deuxième génération contenant les chromosomes humains désirés, une population de micro-cellules qui sont fusionnées avec des cellules ES. La sélection par le G418 permet d'isoler les cellules ES contenant le chromosome humain d'intérêt. Après injection de ces cellules ES ainsi modifiées dans des blastocystes, des souris chimères sont obtenues. Les auteurs se sont intéressés particulièrement à des souris possédant, soit le chromosome 2 , soit le 14 , soit le 22, c'est-à-dire les chromosomes sur lesquels sont situés les locus des gènes d'immunoglobuline $I G G K$, $I G G H$ et $I G G L$. Un très haut niveau d'expression de ces immunoglobulines humaines, témoignant d'un phénomène normal de réarrangement dans le système lymphocytaire murin, peut être observé tout au long de la vie des animaux chimères; les chromosomes humains des souris obtenues se maintiennent donc de manière stable chez des souris transgéniques par ailleurs phénotypiquement normales à l'exception de deux stérilités observées chez des mâles. Cette absence de phénotype et cette stabilité n'allaient pas de soi puisque les souris sont totalement ou partiellement trisomiques: elles contiennent en effet les deux copies d'une paire de chromosomes murins et les régions synténiques correspondantes d'un chromosome humain célibataire. Le passage dans la lignée germinale du chromosome humain n'a été observé que pour un fragment du chromosome 2 , transmis de manière stable par les mâles et les femelles sur plusieurs générations [1]. Il n'est naturellement pas sûr que cette méthode puisse être employée avec n'importe quel chromosome humain, une trisomie chimérique pouvant, selon les cas, entraîner un phénotype plus ou moins anormal. De même, les conséquences sur la méiose de certaines de ces trisomies pourraient empêcher le passage dans la lignée germinale. Dans l'expérience de Tomizuka et al., les conséquences variables d'une trisomie chimérique du 2, du 14 et du 22 pourraient expliquer que seul un fragment du chromosome 2 ait pu être transmis à la lignée germinale. Il n'empêche que ces résultats ouvrent une voie nouvelle pour créer des souris transgéniques comportant des chromosomes humains normaux ou artificiels $\left(m / s \quad n^{\circ} 8 / 9\right.$, vol. 13, p. 1066). Outre l'expression correcte de certains gènes possédant des éléments de structure et de régulation disséminés sur de très grands fragments d'ADN, cette approche devrait également permettre de créer des modèles animaux de maladies humaines avec désordres cytogénétiques, par exemple la trisomie 21 .

[1. Tomizuka K, et al. Nature Genet $1987 ; 16: 133-43$.] 